\title{
An Empirical Comparison of Meta-analysis and Mega-analysis of Individual Participant Data for Identifying Gene-Environment Interactions
}

\author{
Yun Ju Sung, ${ }^{1 *}$ Karen Schwander, ${ }^{1}$ Donna K. Arnett, ${ }^{2}$ Sharon L.R. Kardia, ${ }^{3}$ Tuomo Rankinen, ${ }^{4}$ Claude Bouchard, ${ }^{4}$ \\ Eric Boerwinkle, ${ }^{5}$ Steven C. Hunt, ${ }^{6}$ and Dabeeru C. Rao ${ }^{1}$
}

\begin{abstract}
${ }^{1}$ Division of Biostatistics, Washington University School of Medicine in St. Louis, St. Louis, Missouri, United States of America; ${ }^{2}$ Department of Epidemiology, School of Public Health, University of Alabama at Birmingham, Birmingham, Alabama, United States of America; ${ }^{3}$ Department of Epidemiology, School of Public Health, University of Michigan, Ann Arbor, Michigan, United States of America; ${ }^{4}$ Human Genomics Laboratory, Pennington Biomedical Research Center, Baton Rouge, Louisiana, United States of America; ${ }^{5}$ Human Genetics Center, School of Public Health, University of Texas Health Science Center at Houston, Houston, Texas, United States of America; ${ }^{6}$ Cardiovascular Genetics, University of Utah, Salt Lake City, Utah, United States of America
\end{abstract}

Received 24 November 2013; Revised 10 February 2014; accepted revised manuscript 28 February 2014.

Published online 9 April 2014 in Wiley Online Library (wileyonlinelibrary.com). DOI 10.1002/gepi.21800

\begin{abstract}
For analysis of the main effects of SNPs, meta-analysis of summary results from individual studies has been shown to provide comparable results as "mega-analysis" that jointly analyzes the pooled participant data from the available studies. This fact revolutionized the genetic analysis of complex traits through large GWAS consortia. Investigations of gene-environment $(\mathrm{G} \times \mathrm{E})$ interactions are on the rise since they can potentially explain a part of the missing heritability and identify individuals at high risk for disease. However, for analysis of gene-environment interactions, it is not known whether these methods yield comparable results. In this empirical study, we report that the results from both methods were largely consistent for all four tests; the standard 1 degree of freedom (df) test of main effect only, the $1 \mathrm{df}$ test of the main effect (in the presence of interaction effect), the $1 \mathrm{df}$ test of the interaction effect, and the joint $2 \mathrm{df}$ test of main and interaction effects. They provided similar effect size and standard error estimates, leading to comparable $P$-values. The genomic inflation factors and the number of SNPs with various thresholds were also comparable between the two approaches. Mega-analysis is not always feasible especially in very large and diverse consortia since pooling of raw data may be limited by the terms of the informed consent. Our study illustrates that meta-analysis can be an effective approach also for identifying interactions. To our knowledge, this is the first report investigating meta-versus mega-analyses for interactions.

Genet Epidemiol 38:369-378, 2014. @ 2014 Wiley Periodicals, Inc.
\end{abstract}

KEY WORDS: gene-environment interactions (GEI); meta-analysis; mega-analysis

\section{Introduction}

Over the past several years, genome wide association studies (GWAS) have identified hundreds of common genetic variants associated with many common complex disease traits (http://www.genome.gov), thereby accelerating the progress in the genetic dissection of complex human disease traits. The confidence and funding invested in GWAS approaches have led to unprecedented levels of collaboration, spawning a number of highly productive consortia such as the CHARGE (Cohorts for Heart and Aging Research in Genetic Epidemiology) [Psaty et al., 2009], GIANT (Genetic Investigation of Anthropometric Traits) [Willer et al., 2009], and ICBP (International Consortium of Blood Pressure) [International Consortium for Blood Pressure Genome-Wide Association et al., 2011]. It is standard practice in these consortia to perform

Supporting Information is available in the online issue at wileyonlinelibrary.com.

* Correspondence to: Dr. Yun Ju Sung, Division of Biostatistics, Washington University School of Medicine, 660 South Euclid Avenue, Campus Box 8067, St. Louis, Missouri 63110, United States of America. E-mail: yunju@wubios.wustl.edu meta-analysis that combines the summary results from multiple studies for the same phenotype of interest [de Bakker et al., 2008]. As recently reviewed [Evangelou and Ioannidis, 2013], meta-analysis provides an efficient and practical strategy for overcoming the limitations of power that can compromise any individual study.

However, these hundreds of successfully identified genetic variants typically have very subtle effects, thus explaining only a fraction of the heritability of most complex traits [Manolio et al., 2009]. It is increasingly recognized that the nearexclusive focus on main effects has become a barrier to the identification of additional genes underlying these disease traits. Increasingly greater emphasis is being placed in recent years on gene-environment $(\mathrm{G} \times \mathrm{E})$ interaction analyses, also relying on meta-analysis in large consortia. The identification of $\mathrm{G} \times \mathrm{E}$ interactions is important for many reasons [Le Marchand and Wilkens, 2008; Thomas, 2010a]. G×E interactions or more complex pathways involving multiple genes and environments can potentially explain at least part of the missing heritability [Eichler et al., 2010; Manolio et al., 
2009; McCarthy and Hirschhorn, 2008; Visscher et al., 2012]. They can further elucidate the biological networks underlying complex disease risk and enable "profiling" of individuals at highest risk for disease. Although identifying $\mathrm{G} \times \mathrm{E}$ interactions is important, it requires larger sample sizes than those needed to identify genetic main effects alone [Thomas, $2010 \mathrm{~b}$ ]. Therefore, it is important to have very large sample sizes. Meta-analysis that has been useful for identifying genetic main effects will be even more useful for identifying $\mathrm{G} \times \mathrm{E}$ interactions.

To increase sample sizes, collaborative groups are increasingly collating the raw individual participant data from studies that are able to participate and jointly analyze the pooled individual-level data (such analysis is referred to here as mega-analysis). This approach, also known as meta-analysis of individual patient data (IPD) in statistical literature [Stewart and Clarke, 1995], is regarded as the gold standard in randomized clinical trials (e.g., see [Chalmers, 1993]). For example, a general framework for this IPD meta-analysis for clinical trials has been described by Higgins et al. [Higgins et al., 2001]. However, meta-analysis that combines results from the participating studies has several advantages as compared to mega-analysis. First, obtaining individual level data is challenging and limited by the terms of the informed consent within each study. Second, integration of very large genotype and phenotypic data sets from different studies is time-consuming and poses additional challenges with data management, storage, and harmonization issues. Third, meta-analysis allows for analyses of individual studies to account for local population substructure, relationships among subjects, study-specific covariates, and other ascertainmentrelated issues that may be optimally dealt with within each study. Reviews on advantages and disadvantages of each approach can be also found elsewhere [Steinberg et al., 1997; Stewart and Tierney, 2002].

Although mega-analysis is arguably more powerful than meta-analysis of separate results, in so far as analysis of main effects is concerned, it has been shown that meta-analysis also yields highly comparable results. Olkin and Sampson [Olkin and Sampson, 1998] showed that, for comparing treatments with respect to a continuous outcome in clinical trials, metaanalysis is equivalent to mega-analysis if the treatment effects and error variances are constant across trials. The equivalence has been extended even if the error variances are different across trials [Mathew and Nordström, 1999]. Lin and Zeng theoretically proved asymptotic equivalence between metaand mega-analyses when the effect sizes are the same for all studies [Lin and Zeng, 2010b]. This was further illustrated empirically by combining results from two phases of data [Lin and Zeng, 2010a].

However, for analysis of $\mathrm{G} \times \mathrm{E}$ interaction effects, it is unknown whether meta- and mega-analyses yield comparable results. In this paper, we addressed this issue using empirical data. Using data from the four studies, we analyzed each study separately and meta-analyzed the results using METAL [Willer et al., 2010]. We also combined the raw phenotypic and GWAS data from all four studies and analyzed the pooled
Table 1. Summary statistics for the four studies

\begin{tabular}{lcccc}
\hline & FHS & GENOA & HERITAGE & HyperGEN \\
\hline \# Subjects & 6,686 & 1,420 & 469 & 1,216 \\
\% Male & $46.6 \%$ & $44.9 \%$ & $48.6 \%$ & $49.7 \%$ \\
Age (mean \pm SD) & $49.1 \pm 13.6$ & $54.7 \pm 10.7$ & $36.9 \pm 14.2$ & $49.5 \pm 13.8$ \\
BMI (mean \pm SD) & $27.4 \pm 5.4$ & $30.4 \pm 6.2$ & $26.0 \pm 5.0$ & $29.3 \pm 5.9$ \\
$\%$ on Anti-HT Meds & $19.0 \%$ & $55.7 \%$ & $0.0 \%$ & $46.0 \%$ \\
SBP (mean \pm SD) & $120.4 \pm 16.4$ & $133.5 \pm 19.6$ & $116.5 \pm 11.2$ & $123.2 \pm 19.2$ \\
Pack Years (mean \pm SD) & $9.9 \pm 17.6$ & $13.6 \pm 21.6$ & $5.0 \pm 10.9$ & $7.2 \pm 16.3$ \\
\hline
\end{tabular}

Values shown represent mean value \pm the standard deviation or percentage. FHS, Framingham Heart Study; GENOA, genetic epidemiology network of arteriopathy; HERITAGE, health, risk factors, exercise training, and genetics; HyperGEN, hypertension genetic epidemiology network.

data (i.e., mega-analysis). In addition to the standard 1 degree of freedom (df) test of interaction effect, we used the joint $2 \mathrm{df}$ test of main and interaction effects by Kraft et al. [Kraft et al., 2007] and the joint meta-analysis by Manning et al. [Manning et al., 2011].

\section{Study Samples}

We used data for European American participants from four studies presented in Table 1: the Framingham Heart Study (FHS), GENOA, HERITAGE, and HyperGEN. All four studies obtained informed consent from participants and approval from the appropriate institutional review boards. All four studies have participated in several GWAS consortia publications (based on main effects), and therefore much is already known about them. Our analysis was restricted to a total of 131,880 imputed SNPs on chromosomes 16 and 18 , for which preliminary analysis of $\mathrm{G} \times \mathrm{E}$ interactions in the FHS data yielded significant results. Imputation for these SNPs was performed using MACH [Li et al., 2010] using the reference panels of the HapMap Phase II data with European ancestry subjects.

The Framingham Heart Study (FHS) is a longitudinal family-based study for identifying the factors that contribute to cardiovascular disease, sponsored by the National Heart, Lung, and Blood Institute (NHLBI). FHS began in 1948 with the recruitment of an original cohort of 5,209 men and women who were 28-62 years of age at entry [Dawber et al., 1951]. Clinic examinations took place approximately every 2 years. In 1971, a second generation of study participants, 5,124 children and spouses of children of the original cohort were enrolled [Feinleib et al., 1975]. Clinic examinations took place approximately every 4 years. Enrollment of the third-generation cohort of 4,095 children of offspring cohort participants began in 2002 [Splansky et al., 2007]. In this analysis, we used the FHS SNP Health Association Resource (SHARe) data, as obtained through the database of Genotypes and Phenotypes ( $\mathrm{dbGaP})$. Our analysis was restricted to cross-sectional data on 6,686 European American subjects involving all three generations with GWAS data.

The Genetic Epidemiology Network of Arteriopathy (GENOA) is a family-based study of hypertension and diabetes (e.g., see [Daniels et al., 2004]). GENOA recruited 
sibships with at least two hypertensive siblings and any additional available siblings, regardless of hypertension status. In total, it has collected data on over 5,000 European-American, African-American, and Hispanic-American subjects, from Minnesota, Mississippi, and Texas, respectively. GENOA subjects were brought in for a complete physical examination and questionnaire. We used the 1,420 European American subjects with GWAS data in this analysis.

The health, risk factors, exercise training, and genetics (HERITAGE) family study was designed to evaluate the role of genetic and nongenetic factors in cardiovascular, metabolic, and hormonal responses to aerobic exercise training [Bouchard et al., 1995]. Extensive data, including body composition, cardiovascular risk factors, and lifestyle habits, were gathered on approximately 800 subjects in over 200 families, both before and after 20 weeks of supervised training. We used 469 European American subjects with GWAS data in this analysis.

The Hypertension Genetic Epidemiology Network (HyperGEN) is a multicenter family-based study for investigating the genetic causes of hypertension and related conditions [Williams et al., 2000]. HyperGEN recruited African American and European American participants at five field centers. It recruited sibships with at least two hypertensive siblings, nonmedicated offspring of these hypertensive siblings, and an age-matched random sample. Subjects were brought into the clinic for an exam, and data were collected from questionnaires, a physical exam, and blood and urine samples. In total, HyperGEN collected data from 2,471 European American subjects and 2,300 African-American subjects. We used the 1,216 European American subjects with GWAS data in this analysis.

\section{Statistical Analyses}

To compare meta-analysis and mega-analysis, we considered four analysis options. First, as most GWAS use a maineffect-only analysis, we also performed a main-effect-only analysis. The expected response trait (Y) has the regression model

$$
E[Y]=\delta_{0}+\delta_{e} E+\delta_{g} G,
$$

where $\delta_{e}$ is the environmental main effect and $\delta_{g}$ is the genetic main effect. We used the Wald test statistic that follows a chisquared distribution with 1 degrees of freedom (df) under the hypothesis $\mathrm{H}_{0}$ : $\delta_{g}=0$ (i.e., testing for the genetic main effect). Second, we followed the standard approach to identify $\mathrm{G} \times \mathrm{E}$ interactions. The regression model is generalized to

$$
E[Y]=\alpha+\beta_{e} E+\beta_{g} G+\beta_{g e} G E,
$$

where $\beta_{e}$ and $\beta_{g}$, respectively, are the environmental and genetic main effects and $\beta_{g e}$ is their multiplicative interaction effect. We used the Wald test statistic that also follows a chisquared distribution with $1 \mathrm{df}$ under the $\mathrm{H}_{0}: \beta_{g e}=0$ (i.e., testing for the $\mathrm{G} \times \mathrm{E}$ interaction effect in the presence of the genetic main effect). Third, we also tested the genetic main effect in the presence of $\mathrm{G} \times \mathrm{E}$ interaction effect by using Wald test statistic that follows a chi-squared distribution with $1 \mathrm{df}$ under the $\mathrm{H}_{0}: \beta_{g}=0$. Finally, we performed the test proposed by Kraft et al. [Kraft et al., 2007] that jointly tests the genetic main and $\mathrm{G} \times \mathrm{E}$ interaction effects using the same regression model as in the second analysis. We used a Wald test statistic that follows a chi-squared distribution with $2 \mathrm{df}$ under the $\mathrm{H}_{0}: \beta_{g}=\beta_{g e}=0$. This Wald test statistic is based on estimates of $\beta_{g}$ and $\beta_{g e}$ and their corresponding $2 \times 2$ covariance matrix. The regression models for mega-analysis of pooled individual data were analogous except that they included study-specific intercepts (i.e., $\alpha_{1}-\alpha_{4}$ ).

To combine the results from the four studies, we performed fixed effects meta-analysis. We used the inversevariance weighting method in METAL [Willer et al., 2010] by computing inverse-variance-weighted coefficients. It is straightforward to combine the $1 \mathrm{df}$ results from three analysis options: main-effect-only analysis, interaction effect, and main effect in the presence of interaction effect. Manning et al. [Manning et al., 2011] developed the joint meta-analysis and modified METAL [Willer et al., 2010] to combine the joint $2 \mathrm{df}$ tests across multiple studies. The joint meta-analysis provides inference on the genetic main effect and interaction effect pooled across all studies. To combine the joint 2 df results (estimates of $\beta_{g}, \beta_{g e}$ and their corresponding $2 \times 2$ covariance matrix) from these four studies, we used a modified version of METAL that implements this joint $2 \mathrm{df}$ meta-analysis by Manning et al. [Manning et al., 2011].

Fixed effects meta-analysis that we used is the most popular approach in GWAS consortia and is shown to be the most powerful for prioritizing and discovering SNPs [Pfeiffer et al., 2009]. In particular, fixed effects models have an advantage over random effects models in terms of increased power for discovery [Pereira et al., 2009]. However, because fixed effects meta-analysis assumes that true effect size is constant across the studies, we examined whether there was evidence of heterogeneity among studies using $P$-value based on Cochran's Q-test [Cochran, 1954] for each SNP for all four analysis options. We also examined the $I^{2}$ index that quantifies the proportion of total variation due to heterogeneity (i.e., between-study variance) [Higgins and Thompson, 2002].

Increased Type I error due to inflation of test statistics in $\mathrm{G} \times \mathrm{E}$ interactions can be a concern as recently shown [Voorman et al., 2011]. We plotted quantile-quantile (QQ) plots and computed the genomic inflation factor $\lambda_{\mathrm{GC}}$, the degree of inflation of the median test statistic, for all four analysis options. We also computed the genomic controlled $P$-values by dividing test statistics by $\lambda_{\mathrm{GC}}$, as they are widely used to correct for minor substructure problems [Ganesh et al., 2009]. In addition, we performed genomic controlled meta-analysis (also using METAL), where genomic control correction was applied before combining study-specific results.

To compare meta-analysis and mega-analysis in the context of $\mathrm{G} \times \mathrm{E}$ interactions, we analyzed systolic blood pressure (SBP) and used pack-years of smoking as an interacting covariate. In addition, we included age, sex, body mass index (BMI), and use of anti-hypertensive medications as common covariates. Because all four studies are family studies 
Table 2. The number of SNPs (out of 131,880 SNPs) and $P^{2}$ index across multiple ranges of heterogeneity $P$-values (HetP) based on Cochran's $\mathbf{Q}$-test for heterogeneity in our meta-analysis

\begin{tabular}{|c|c|c|c|c|c|c|c|}
\hline \multirow[t]{2}{*}{ Range of HetP } & \multicolumn{2}{|c|}{ 1DF main only } & \multicolumn{2}{|c|}{ 1DF main } & \multicolumn{2}{|c|}{ 1DF interaction } & \multirow{2}{*}{$\begin{array}{l}\text { 2DF joint main and int. } \\
\text { SNPs count }(\%)\end{array}$} \\
\hline & SNPs count $(\%)$ & $\begin{array}{l}I^{2} \text { index mean } \\
(\min , \max )\end{array}$ & SNPs count $(\%)$ & $\begin{array}{l}I^{2} \text { index mean } \\
\quad(\min , \max )\end{array}$ & SNPs count $(\%)$ & $\begin{array}{l}I^{2} \text { index mean } \\
\quad(\min , \max )\end{array}$ & \\
\hline HetP $\geq 0.1$ & $119,421(90.6)$ & $0.8(0,20)$ & $119,776(90.8)$ & $0.8(0,20)$ & $103,726(78.7)$ & $1.3(0,20)$ & $109,302(82.9)$ \\
\hline $0.05 \leq \mathrm{HetP}<0.1$ & $6,047(4.6)$ & $27.6(20,36)$ & $6,084(4.6)$ & $27.6(20,36)$ & $11,264(8.5)$ & $28.0(20,36)$ & $9,566(7.3)$ \\
\hline HetP $<0.05$ & $6,412(4.9)$ & $48.2(36,77.5)$ & $6,020(4.6)$ & $48.0(36,76.5)$ & $16,890(12.8)$ & $50.8(36,81.9)$ & $13,012(9.9)$ \\
\hline All & & $4.3(0,77.5)$ & & $4.2(0,76.5)$ & & $9.9(0,81.9)$ & \\
\hline
\end{tabular}

$I^{2}$ index for the $2 \mathrm{df}$ joint main and interaction test is unavailable.

in which relatedness must be taken into account, we used ProbABEL [Aulchenko et al., 2010] and GenABEL/MixABEL [Aulchenko et al., 2007] for each cohort study and megaanalysis of pooled individual participant data.

\section{Results}

Table 1 presents select characteristics of the four studies. Roughly half of the subjects are female within each study. The subjects in the HERITAGE tended to be younger, relatively leaner and low pack years with no anti-hypertensive medications (e.g., hypertension was an exclusion criterion). In contrast, the subjects in GENOA were older and had high pack years and higher SBP with over 55\% on anti-hypertensive medications. The HyperGEN study has characteristics similar to GENOA. The FHS has intermediate values for SBP, medication use and pack years. We found that meta-analysis of interaction results had more SNPs with evidence of heterogeneity across studies.

Table 2 presents the number of SNPs and $I^{2}$ index across multiple ranges of heterogeneity $P$-values based on Cochran's $Q$-test for heterogeneity. For main effect only analysis, heterogeneity $P$-values roughly followed a uniform distribution; about $5 \%$ of SNPs had heterogeneity $P$-values below 0.05 . In contrast, meta-analysis for the interaction effect encountered the greatest heterogeneity among studies (13\% of the SNPs had heterogeneity $P$-values $<0.05)$. The $2 \mathrm{df}$ test of joint main and interaction effects provided $10 \%$ heterogeneous results. Values of $I^{2}$ index also provided evidence of higher heterogeneity in the interaction analysis $\left(I^{2}\right.$ values of $25,50,75$ are considered low, moderate, high heterogeneity, respectively). $I^{2}$ index ranged from 0 to 77.5 with a mean value of 4.3 for the main effect only analysis, whereas it ranged from 0 to 81.9 with a mean value of 9.9 for the interaction analysis.

We found that the results between meta- and megaanalyses were largely consistent. In particular, as shown in Figure 1, the two analysis approaches provided similar effect sizes and standard error (SE) estimates. Effect sizes between the two approaches had a correlation of $0.95,0.94$, and 0.96 for main effect only $\left[\delta_{g}\right.$ in equation $\left.(1)\right]$, main effect $\left[\beta_{g}\right.$ in equation (2)], and interaction effect $\left[\beta_{g e}\right.$ in equation (2)] estimates, respectively. When the results were restricted to SNPs with heterogeneous effect sizes across studies (i.e., $P$-values of Cochran's $Q$-test $<0.05)$, there was larger variation in the meta- and mega-analysis results, leading to smaller correla- tions (as expected). Finally, SE estimates for all three effect sizes were almost identical (with correlations of 0.999 ).

Figure 2 presents $-\log (\mathrm{p})$ values between meta- and megaanalyses for all four analysis options: main-effect-only analysis, main effect in the presence of the interaction effect, interaction effect in the presence of the main effect, and 2 df joint tests of main and interaction effects. Supplementary Table 1 presents summary statistics of $-\log (\mathrm{p})$ differences between mega- and meta-analyses. Surprisingly, the joint $2 \mathrm{df}$ test provided the highest overall correlation (0.91); the correlations between the two methods across all SNPs were identical to the correlations across homogeneous SNPs only (up to two decimal places). Overall, although mega-analysis provided slightly better results as expected, both methods yielded very similar results for the most promising SNPs (with the smallest $P$ values). For these promising SNPs, heterogeneity $P$-values were greater than 0.05 , indicating their effects are homogenous across studies. High concordance of the smallest $P$ values between mega- and meta-analysis methods is particularly noteworthy since they are the most important discoveries for further follow up.

When the effect sizes are constant across studies, the metaanalysis method using inverse-variance weighting is shown to be optimal [Zhou et al., 2011]. In particular, weights proportional to the square root of study sample sizes can be suboptimal when the trait variance differs across studies. In addition to the weighting method, we also performed another commonly used approach that converts the direction of effect and $P$-value observed in each study into a signed $\mathrm{Z}$-score; these $\mathrm{Z}$-scores are then combined across studies in a weighted sum, with weights proportional to the square-root of the sample size for each study. Although trait variance of SBP differs somewhat, as shown in Table 1, the results between the inverse-variance weighting method and the sample size weighted signed $\mathrm{Z}$ score method were remarkably similar, providing a correlation of 0.98 .

Table 3 presents genomic inflation factor $\lambda_{\mathrm{GC}}$ for all four analysis options. QQ plots for meta- and mega-analyses are shown in Figure 3 (study-specific QQ plots are shown in Supplementary Fig. 1). We found that both meta- and megaanalyses behaved similarly. Main effect analysis had no inflation of Type I error. However, $1 \mathrm{df}$ test of interaction effect had high inflation of type I error $\left(\lambda_{\mathrm{GC}}=1.45\right.$ for both metaand mega-analyses). The genomic controlled meta-analysis (GC-Meta), where genomic control correction was applied before combining study-specific results, removed most of the 

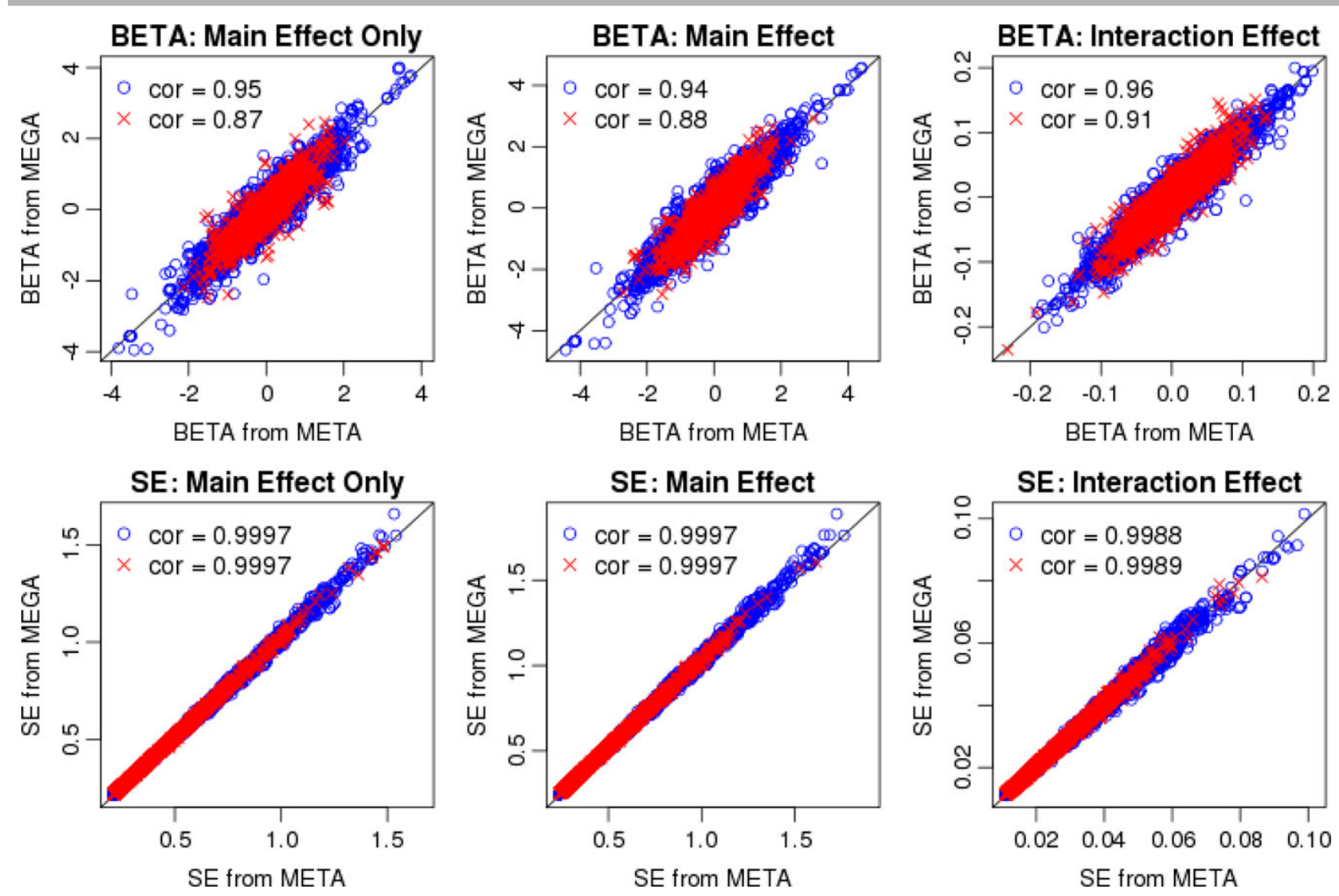

Figure 1. Scatterplots showing effect sizes (1st row) and standard errors (2nd row) from mega-analysis versus meta-analysis. The main effect only BETA (1st column) corresponds to $\delta_{g}$ in equation (1). The main effect BETA (2nd column) and interaction effect BETA (3rd column) correspond to $\beta_{g}$ and $\beta_{g e}$ in equation (2), respectively. Red crosses are SNPs with heterogeneous effect sizes across studies (i.e., $P$-value of Cochran's $Q$-test $<$ $0.05)$, and blue circles are SNPs with $P$-value of Cochran's $Q$-test $\geq 0.05$. The correlations between the two methods across all SNPs were identical to the correlations across blue circles (up to two decimal places). The black diagonal line indicates where the values on the two axes are equal.

inflation $\left(\lambda_{\mathrm{GC}}=1.02\right)$. The $2 \mathrm{df}$ joint test of main and interaction effects had lower levels of inflation of type I error $\left(\lambda_{\mathrm{GC}}=1.15\right.$ and 1.20 for meta- and mega-analysis, respectively), which was also removed by GC-Meta $\left(\lambda_{\mathrm{GC}}=0.99\right)$. As shown in Table 4, the proportion of SNPs with $P$-value $<0.05$ stayed at $5 \%$ with genomic control correction. We note that, as the primary purpose of this investigation is to evaluate the comparability of meta- versus mega-analysis, inflation of type I error rate in $\mathrm{G} \times \mathrm{E}$ analyses is in itself not as relevant as whether such inflation, when it exists, is also comparable between the two approaches.

For mega-analysis, we also used the robust option in the ProABEL suite (results shown in Supplementary Fig. 2) following a recent recommendation [Voorman et al., 2011]. This robust option is based on the "sandwich" estimate for variance, which is known to provide robust and asymptotically consistent estimator under miss-specified models [Huber, 1967; Liang and Zeger, 1986; White, 1980]. Because the robust option in ProbABEL is currently applicable to populationbased data, this ignores familial correlation. As shown in Supplementary Figure 2, the $P$-values from this robust option were similar to the genomic controlled $P$-values from the mega-analysis (Mega-GC).
We compared the performance of the four analyses in terms of the evidence for association, using the genomic controlled mega-analysis (Mega-GC) results. Figure 4 presents the scatterplots of the results $[-\log (p)$ values] across these four analysis options. As shown in Table 5, the number of SNPs with $P$-value $<1 \times 10^{-4}$ was $24,2,18$, and 35 from main effect only, main effect (in the presence of interaction), interaction effect (in the presence of main effect), and the $2 \mathrm{df}$ joint test, respectively. They are plotted as red, green, blue, and cyan points in Figure 4 . The 18 blue SNPs (with $P$-value < $1 \times 10^{-4}$ from the interaction effect) also had $P$-value $<1 \times$ $10^{-4}$ from the $2 \mathrm{df}$ joint test; 17 additional SNPs with $P$-value $<1 \times 10^{-5}$ from the $2 \mathrm{df}$ joint test are plotted as cyan points. Table 5 presents the effect sizes and standard errors (SE) for a subset of these SNPs, one SNP selected from each linkage disequilibrium (LD) block, as our results were based on 131,880 imputed SNPs. We found that the genetic main and interaction effects were in the opposite direction for every SNP in group 2. For these SNPs, the main effect only analysis (without interaction) provided a much smaller main effect estimate, leading to no evidence of association. This phenomenon is well known as Simpson's paradox in the statistics literature [Simpson, 1951; Wagner, 1982]. We found that 

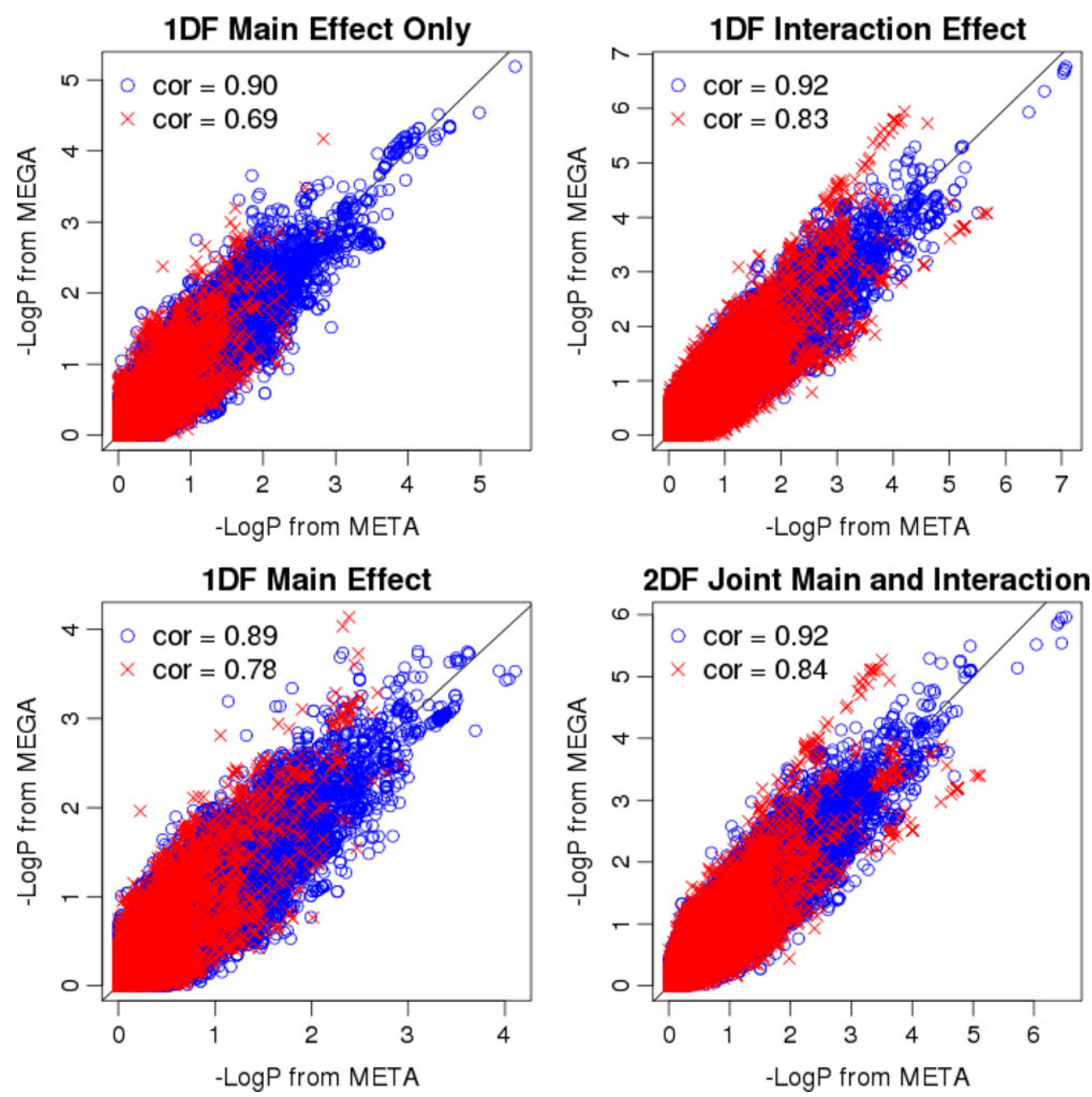

Figure 2. Scatterplots showing - $\log (p)$ values from mega-analysis versus meta-analysis for the four analysis options: $1 \mathrm{df}$ test of the main effect only (without interaction effect) (top left), $1 \mathrm{df}$ test of main effect in the presence of interaction effect (bottom left), $1 \mathrm{df}$ test of interaction effect in the presence of the main effect (top right), and $2 \mathrm{df}$ joint test of the main and interaction effects (bottom right). Red crosses are SNPs with heterogeneity effect sizes across studies (i.e., $P$-value of Cochran's $Q$-test $<0.05$ ), and blue circles are SNPs with $P$-value of Cochran's $Q$-test $\geq$ 0.05. The correlations between the two methods across all SNPs were identical to the correlations across blue circles (up to two decimal places). The black diagonal line indicates where -log $(p)$ values from meta- and mega-analyses are equal.

Table 3. Genomic control $\lambda_{\mathrm{GC}}$ values for study-specific analysis, mega-anlysis, and meta-analysis

\begin{tabular}{lcccc}
\hline & $\begin{array}{c}\text { 1DF } \\
\text { main } \\
\text { only }\end{array}$ & $\begin{array}{c}\text { 1DF } \\
\text { main }\end{array}$ & $\begin{array}{c}\text { 1DF } \\
\text { interaction }\end{array}$ & $\begin{array}{c}\text { 2DF joint } \\
\text { main and } \\
\text { int. }\end{array}$ \\
\hline $\begin{array}{l}\text { Study-specific analysis: } \\
\quad \text { FHS }\end{array}$ & 0.905 & 0.927 & 1.466 & 1.161 \\
GENOA & 0.998 & 1.108 & 1.228 & 1.117 \\
HERITAGE & 1.047 & 0.954 & 1.518 & 1.238 \\
HyperGEN & 1.015 & 0.933 & 1.549 & 1.234 \\
$\quad$ Mega-analysis: & 1.036 & 0.988 & 1.451 & 1.204 \\
Meta-analysis: & & & & \\
$\quad \begin{array}{l}\text { No genomic control correction } \\
\text { Genomic control correction }\end{array}$ & 0.976 & 0.927 & 1.447 & 1.154 \\
\hline
\end{tabular}

QQ-plots for study-specific analysis are shown in Supplementary Figure 1. QQ-plots for meta- and mega-analyses are shown in Figure 3.

the main and interaction effects were in the same direction for every SNPs in groups 1 and 3. Although this evaluation was based on results from the mega-analysis, as they are considered a gold standard, results based on meta-analysis were almost identical (which is not surprising given the high concordance between mega- and meta-analysis).

\section{Discussion}

Meta-analysis has become the most effective approach in GWAS for utilizing large sample sizes available from multiple studies and a standard practice in most consortia, since each study can analyze its own data optimally by customizing data adjustments as needed [Evangelou and Ioannidis, 2013]. Increasingly greater emphasis is being placed on $\mathrm{G} \times \mathrm{E}$ interaction analyses, also relying on meta-analysis in large consortia [Aschard et al., 2012]. Mega-analysis is not readily feasible owing to several challenges including limitations of the informed consent for sharing raw data, data management, and data harmonization for combining multiple huge 

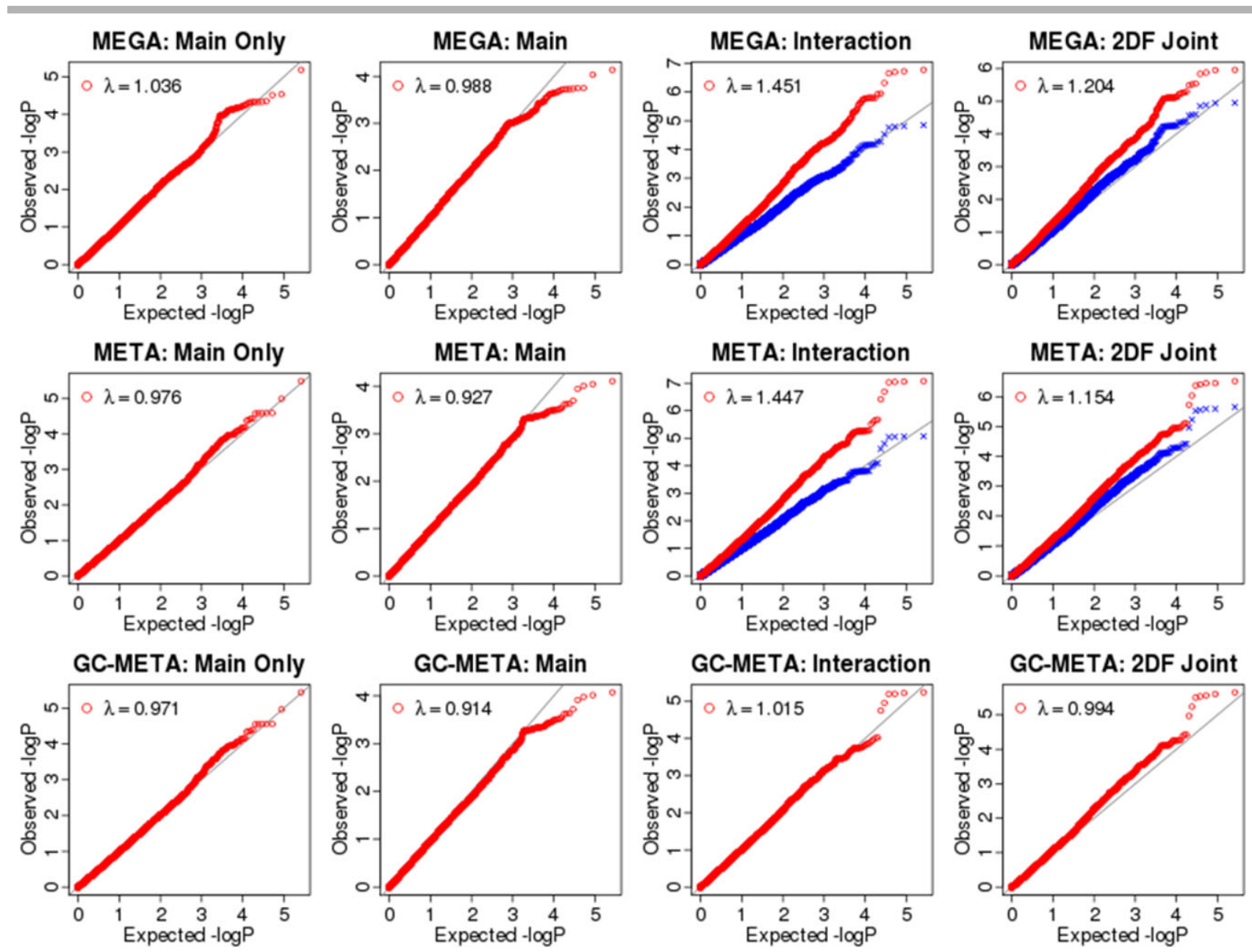

Figure 3. Quantile-quantile (00) plots for the four analysis options: main effect only analysis (1st column), main effect in the presence of interaction (2nd column), interaction in the presence of main effect (3rd column), and $2 \mathrm{df}$ joint test of main and interaction effects (4th column). The red circle points are $P$-values and the blue cross points are genomic controlled $P$-values. The genomic control value for the blue cross points is 1. Study-specific 00 plots are shown in Supplementary Figure 1.

Table 4. The number of SNPs with $P$-values below thresholds $\left(0.05\right.$ and $\left.1 \times 10^{-4}\right)$ out of 131,880 SNPs. Gonomic control (GC) correction preserved type I error

\begin{tabular}{|c|c|c|c|c|c|}
\hline & Analysis & 1DF main only & 1DF main & 1DF interaction & $2 \mathrm{DF}$ joint main and int. \\
\hline \multirow[t]{5}{*}{$P<0.05$} & Mega-analysis & $6,617(6 \%)$ & $5,935(5 \%)$ & $13,584(11 \%)$ & $11,691(9 \%)$ \\
\hline & Meta-analysis & $6,617(6 \%)$ & $5,935(5 \%)$ & $13,584(11 \%)$ & $11,691(9 \%)$ \\
\hline & Mega-GC analysis & $6,896(5 \%)$ & $7,167(5 \%)$ & $6,469(5 \%)$ & $7,529(6 \%)$ \\
\hline & Meta-GC analysis & $6,970(5 \%)$ & $7,024(5 \%)$ & $6,604(5 \%)$ & $7,945(6 \%)$ \\
\hline & GC-Meta-analysis & $6,527(5 \%)$ & $5,710(4 \%)$ & $6,752(5 \%)$ & $7,790(6 \%)$ \\
\hline \multirow[t]{5}{*}{$P<1 \times 10^{-4}$} & Mega-analysis & 37 & 2 & 215 & 108 \\
\hline & Meta-analysis & 20 & 3 & 188 & 118 \\
\hline & Mega-GC analysis & 20 & 2 & 8 & 35 \\
\hline & Meta-GC analysis & 32 & 4 & 9 & 36 \\
\hline & GC-Meta-analysis & 20 & 2 & 8 & 35 \\
\hline
\end{tabular}

For Mega-GC and Meta-GC, GC correction was applied after mega-analysis and meta-analysis, respectively. For GC-Meta, GC correction was applied for each study-specific analysis before meta-analysis.

datasets. All these challenges render meta-analysis as the convenient and expedient method of choice especially because meta-analysis yields results comparable to mega-analysis.

In this paper, we empirically evaluated whether metaanalysis also provides results comparable to mega-analysis for identifying $\mathrm{G} \times \mathrm{E}$ interactions. We found that results from meta- and mega-analyses were largely consistent even for identifying $\mathrm{G} \times \mathrm{E}$ interaction effects. In particular, they provided similar effect size and standard error estimates, leading to similar $P$-values. The number of SNPs significant at various 

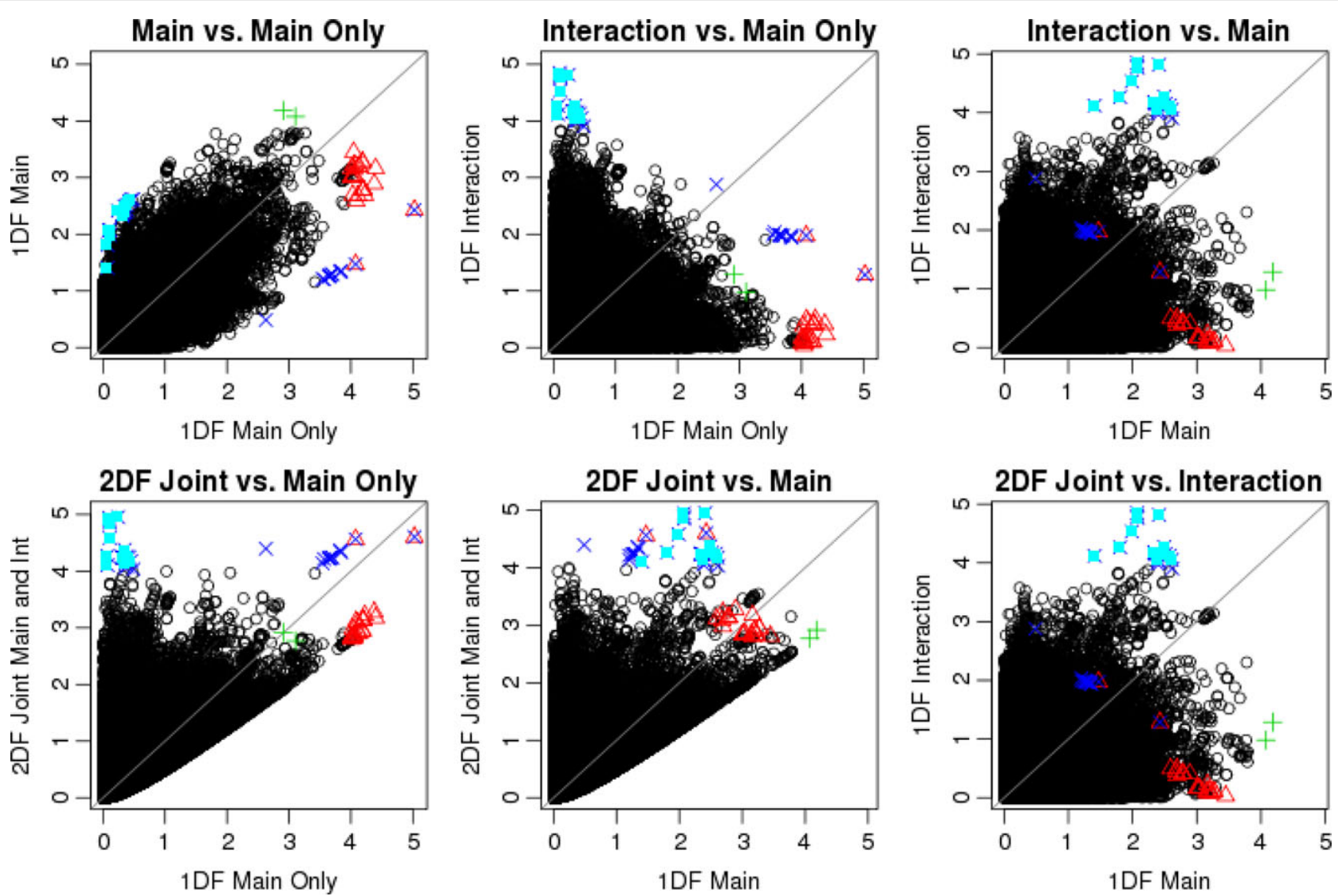

Figure 4. Scatterplots of $-\log (p)$ values across the four analysis options using genomic controlled mega-analysis. Red points are 24 SNPs with $P$-value $<1 \times 10^{-4}$ from the main effect only test. Green points are two SNPs with $P$-value $<1 \times 10^{-4}$ from the main effect test. Blue points are 18 SNPs with $P$-value $<1 \times 10^{-4}$ from the $1 \mathrm{df}$ test of interaction effect (in the presence of main effect). Cyan points are additional 17 SNPs with $P$-value $<1 \times 10^{-4}$ from the $2 \mathrm{df}$ test of joint main and interaction effects.

Table 5. Independent SNPs with $P$-values $<1 \times 10^{-4}$, one SNP selected from each linkage disequilibrium (LD) block from the genomic controlled mega-analysis

\begin{tabular}{|c|c|c|c|c|c|c|c|c|c|c|}
\hline \multirow[b]{2}{*}{ SNP } & \multicolumn{2}{|c|}{ Main only } & \multicolumn{2}{|c|}{ Main } & \multicolumn{2}{|c|}{ Interaction } & \multicolumn{4}{|c|}{ Genomic controlled $P$-values } \\
\hline & Beta & SE & Beta & SE & Beta & SE & Main only & Main & Int & $2 \mathrm{DF}$ \\
\hline \multicolumn{11}{|c|}{ Group 1 (selected from 22 red SNPs in Fig. 4) } \\
\hline rs8050871 & 1.10 & 0.24 & 0.80 & 0.28 & 0.03 & 0.01 & $9.4 \times 10^{-6}$ & 0.004 & 0.05 & $2.5 \times 10^{-5}$ \\
\hline rs4788828 & -1.23 & 0.30 & -1.18 & 0.34 & -0.01 & 0.02 & $6.5 \times 10^{-5}$ & 0.0005 & 0.79 & 0.001 \\
\hline rs948638 & 1.30 & 0.31 & 1.13 & 0.35 & 0.02 & 0.02 & $4.2 \times 10^{-5}$ & 0.001 & 0.38 & 0.0005 \\
\hline rs6499538 & 1.21 & 0.30 & 1.18 & 0.34 & 0.00 & 0.02 & $8.7 \times 10^{-5}$ & 0.0005 & 0.84 & 0.001 \\
\hline rs2247603 & 1.26 & 0.32 & 1.18 & 0.36 & 0.01 & 0.02 & $8.8 \times 10^{-5}$ & 0.0009 & 0.69 & 0.001 \\
\hline rs9930015 & 1.24 & 0.31 & 1.16 & 0.35 & 0.01 & 0.02 & $9.7 \times 10^{-5}$ & 0.0009 & 0.68 & 0.001 \\
\hline \multicolumn{11}{|c|}{ Group 2 (selected from 18 blue SNPs in Fig. 4) } \\
\hline rs733123 & 0.18 & 0.32 & 1.03 & 0.36 & -0.09 & 0.02 & 0.57 & 0.004 & $1.5 \times 10^{-5}$ & $1.1 \times 10^{-5}$ \\
\hline rs9954614 & 0.08 & 0.32 & 0.94 & 0.36 & -0.09 & 0.02 & 0.80 & 0.008 & $1.4 \times 10^{-5}$ & $1.1 \times 10^{-5}$ \\
\hline rs6420435 & -0.21 & 0.27 & -0.92 & 0.31 & 0.07 & 0.01 & 0.45 & 0.003 & $5.3 \times 10^{-5}$ & $4.1 \times 10^{-5}$ \\
\hline rs1558949 & 0.06 & 0.31 & 0.85 & 0.36 & -0.08 & 0.02 & 0.84 & 0.015 & $5.4 \times 10^{-5}$ & $5.4 \times 10^{-5}$ \\
\hline rs11859599 & -0.24 & 0.27 & -0.90 & 0.30 & 0.07 & 0.01 & 0.38 & 0.003 & $7.5 \times 10^{-5}$ & $5.6 \times 10^{-5}$ \\
\hline rs2405214 & 0.05 & 0.23 & -0.54 & 0.26 & 0.06 & 0.01 & 0.85 & 0.039 & $7.6 \times 10^{-5}$ & $7.8 \times 10^{-5}$ \\
\hline \multicolumn{11}{|c|}{ Group 3 (selected from 2 green SNPs and 17 cyan SNPs in Fig. 4) } \\
\hline rs963267 & 0.91 & 0.23 & 0.55 & 0.26 & 0.04 & 0.01 & $8.4 \times 10^{-5}$ & 0.03 & 0.01 & $2.7 \times 10^{-5}$ \\
\hline rs949184 & -1.24 & 0.33 & -0.72 & 0.37 & -0.05 & 0.02 & 0.0002 & 0.05 & 0.01 & $5.7 \times 10^{-5}$ \\
\hline rs9960318 & -1.39 & 0.42 & -1.89 & 0.48 & 0.05 & 0.02 & 0.001 & $6.5 \times 10^{-5}$ & 0.05 & 0.001 \\
\hline
\end{tabular}


thresholds was also comparable between the two approaches. We observed increased type I error due to inflation of test statistics for identifying interactions. To address this issue, we adjusted for genomic control before and after meta-analysis in addition to correction after mega-analysis. Although inflation of type I error rate has been observed for the analysis of $\mathrm{G} \times \mathrm{E}$ interactions, the levels of inflation were fairly comparable between meta- and mega-analyses. Our study illustrates that meta-analysis can be an effective approach for investigating interaction effects also.

To our knowledge, this is the first report investigating metaversus mega-analyses for interactions. The validity of our findings is somewhat limited as it is based on an empirical investigation using a relatively small number of studies (four) that are readily available to us. Therefore, they may not be generalized to all situations. A more comprehensive simulation study covering the various scenarios regarding the number of studies, variation of study sizes, and types of genetic variation, among others, would strengthen our findings.

\section{Acknowledgments}

We thank anonymous reviewers for their constructive and insightful comments, which substantially improved the manuscript. The work was partly supported by NIH Grants HL107552, HL111249, HL118305, HL055673, and HL086694. C.B. is partially funded by the John W. Barton Sr. Chair in Genetics and Nutrition. The authors declare that they have no competing interests.

\section{References}

Aschard H, Lutz S, Maus B, Duell EJ, Fingerlin TE, Chatterjee N, Kraft P, Van Steen K. 2012. Challenges and opportunities in genome-wide environmental interaction (GWEI) studies. Hum Genet 131(10):1591-1613.

Aulchenko YS, Ripke S, Isaacs A, van Duijn CM. 2007. GenABEL: an R library for genome-wide association analysis. Bioinformatics 23(10):1294-1296.

Aulchenko YS, Struchalin MV, van Duijn CM. 2010. ProbABEL package for genomewide association analysis of imputed data. BMC Bioinformatics 11:134

Bouchard C, Leon AS, Rao DC, Skinner JS, Wilmore JH, Gagnon J. 1995. The HERITAGE family study. Aims, design, and measurement protocol. Med Sci Sports Exerc 27(5):721-729.

Chalmers I. 1993. The Cochrane Collaboration: preparing, maintaining and disseminating systematic reviews of the effects of health care. Ann NY Acad Sci 703:156-163.

Cochran WG. 1954. The combination of estimates from different experiments. Biometrics 10(1):101-129.

Daniels PR, Kardia SL, Hanis CL, Brown CA, Hutchinson R, Boerwinkle E, Turner ST, Genetic Epidemiology Network of Arteriopathy study. 2004. Familial aggregation of hypertension treatment and control in the Genetic Epidemiology Network of Arteriopathy (GENOA) study. Am J Med 116(10):676-681.

Dawber TR, Meadors GF, Moore FE, Jr. 1951. Epidemiological approaches to heart disease: the Framingham Study. Am J Public Health Nations Health 41(3): 279-281.

de Bakker PI, Ferreira MA, Jia X, Neale BM, Raychaudhuri S, Voight BF. 2008. Practical aspects of imputation-driven meta-analysis of genome-wide association studies. Hum Mol Genet 17(R2):R122-R128.

Eichler EE, Flint J, Gibson G, Kong A, Leal SM, Moore JH, Nadeau JH. 2010. Missing heritability and strategies for finding the underlying causes of complex disease. Nat Rev Genet 11(6):446-450.

Evangelou E, Ioannidis JP. 2013. Meta-analysis methods for genome-wide association studies and beyond. Nat Rev Genet 14(6):379-389.

Feinleib M, Kannel WB, Garrison RJ, McNamara PM, Castelli WP. 1975. The Framingham Offspring Study. Design and preliminary data. Prev Med 4(4): $518-525$.

Ganesh SK, Zakai NA, van Rooij FJ, Soranzo N, Smith AV, Nalls MA, Chen MH, Kottgen A, Glazer NL, Dehghan A, and others. 2009. Multiple loci influence erythrocyte phenotypes in the CHARGE Consortium. Nat Genet 41(11):1191-1198.
Higgins JP, Thompson SG. 2002. Quantifying heterogeneity in a meta-analysis. Stat Med 21(11):1539-1558.

Higgins JP, Whitehead A, Turner RM, Omar RZ, Thompson SG. 2001. Metaanalysis of continuous outcome data from individual patients. Stat Med 20(15): 2219-2241.

Huber PJ. 1967. The behavior of maximum likelihood estimates under nonstandard conditions. Proc Fifth Berkeley Symp on Math Statist and Prob 1:221-233.

International Consortium for Blood Pressure Genome-Wide Association S, Ehret GB, Munroe PB, Rice KM, Bochud M, Johnson AD, Chasman DI, Smith AV, Tobin MD, Verwoert GC, and others. 2011. Genetic variants in novel pathways influence blood pressure and cardiovascular disease risk. Nature 478(7367):103-109.

Kraft P, Yen YC, Stram DO, Morrison J, Gauderman WJ. 2007. Exploiting geneenvironment interaction to detect genetic associations. Hum Hered 63(2):111-119.

Le Marchand L, Wilkens LR. 2008. Design considerations for genomic association studies: importance of gene-environment interactions. Cancer Epidemiol Biomarkers Prev 17(2):263-267.

Li Y, Willer CJ, Ding J, Scheet P, Abecasis GR. 2010. MaCH: using sequence and genotype data to estimate haplotypes and unobserved genotypes. Genet Epidemiol 34(8):816-834.

Liang KY, Zeger SL. 1986. Longitudinal data-analysis using generalized linear-models. Biometrika 73(1):13-22.

Lin DY, Zeng D. 2010a. Meta-analysis of genome-wide association studies: no efficiency gain in using individual participant data. Genet Epidemiol 34(1):60-66.

Lin DY, Zeng D. 2010b. On the relative efficiency of using summary statistics versus individual-level data in meta-analysis. Biometrika 97(2):321-332.

Manning AK, LaValley M, Liu CT, Rice K, An P, Liu Y, Miljkovic I, Rasmussen-Torvik L, Harris TB, Province MA, and others. 2011. Meta-analysis of gene-environment interaction: joint estimation of SNP and SNP x environment regression coefficients. Genet Epidemiol 35(1):11-18.

Manolio TA, Collins FS, Cox NJ, Goldstein DB, Hindorff LA, Hunter DJ, McCarthy MI, Ramos EM, Cardon LR, Chakravarti A, and others. 2009. Finding the missing heritability of complex diseases. Nature 461(7265):747-753.

Mathew T, Nordström K. 1999. On the equivalence of meta-analysis using literature and using individual patient data. Biometrics 55(4):1221-1223.

McCarthy MI, Hirschhorn JN. 2008. Genome-wide association studies: potential next steps on a genetic journey. Hum Mol Genet 17(R2):R156-R165.

Olkin I, Sampson A. 1998. Comparison of meta-analysis versus analysis of variance of individual patient data. Biometrics 54(1):317-322.

Pereira TV, Patsopoulos NA, Salanti G, Ioannidis JP. 2009. Discovery properties of genome-wide association signals from cumulatively combined data sets. Am J Epidemiol 170(10):1197-1206.

Pfeiffer R, Gail M, Pee D. 2009. On combining data from genome-wide association studies to discover disease-associated SNPs. Stat Sci 24(4):547-560.

Psaty BM, O'Donnell CJ, Gudnason V, Lunetta KL, Folsom AR, Rotter JI, Uitterlinden AG, Harris TB, Witteman JC, Boerwinkle E, and others. 2009. Cohorts for heart and aging research in genomic epidemiology (CHARGE) consortium: design of prospective meta-analyses of genome-wide association studies from 5 cohorts. Circ Cardiovasc Genet 2(1):73-80.

Simpson EH. 1951. The interpretation of interaction in contingency tables. J Royal Stat Soc Series B 13(2):238-241.

Splansky GL, Corey D, Yang Q, Atwood LD, Cupples LA, Benjamin EJ, D’Agostino RB, Sr., Fox CS, Larson MG, Murabito JM, and others. 2007. The third generation cohort of the national heart, lung, and blood institute's framingham heart study: design, recruitment, and initial examination. Am J Epidemiol 165(11): $1328-1335$.

Steinberg KK, Smith SJ, Stroup DF, Olkin I, Lee NC, Williamson GD, Thacker SB. 1997. Comparison of effect estimates from a meta-analysis of summary data from published studies and from a meta-analysis using individual patient data for ovarian cancer studies. Am J Epidemiol 145(10):917-925.

Stewart LA, Clarke MJ. 1995. Practical methodology of meta-analyses (overviews) using updated individual patient data. Cochrane Working Group. Stat Med 14(19):20572079.

Stewart LA, Tierney JF. 2002. To IPD or not to IPD?: advantages and disadvantages of systematic reviews using individual patient data. Eval Health Professions 25(1):7697.

Thomas D. 2010a. Gene-environment-wide association studies: emerging approaches. Nat Rev Genet 11(4):259-272.

Thomas D. 2010b. Gene-environment-wide association studies: emerging approaches. Nat Rev Genet 11(4):259-272.

Visscher PM, Brown MA, McCarthy MI, Yang J. 2012. Five years of GWAS discovery. Am J Hum Genet 90(1):7-24.

Voorman A, Lumley T, McKnight B, Rice K. 2011. Behavior of QQ-plots and genomic control in studies of gene-environment interaction. PLoS One 6(5):e19416.

Wagner CH. 1982. Simpson's Paradox in real life. Am Stat 36(1):46-48.

White H. 1980. A heteroskedasticity-consistent covariance-matrix estimator and a direct test for heteroskedasticity. Econometrica 48(4):817-838. 
Willer CJ, Li Y, Abecasis GR. 2010. METAL: fast and efficient meta-analysis of genomewide association scans. Bioinformatics 26(17):2190-2191.

Willer CJ, Speliotes EK, Loos RJ, Li S, Lindgren CM, Heid IM, Berndt SI, Elliott AL, Jackson AU, Lamina C, and others. 2009. Six new loci associated with body mass index highlight a neuronal influence on body weight regulation. Nat Genet 41(1):25-34.
Williams RR, Rao DC, Ellison RC, Arnett DK, Heiss G, Oberman A, Eckfeldt JH, Leppert MF, Province MA, Mockrin SC, and others. 2000. NHLBI family blood pressure program: methodology and recruitment in the HyperGEN network. Hypertension genetic epidemiology network. Ann Epidemiol 10(6):389-400.

Zhou B, Shi J, Whittemore AS. 2011. Optimal methods for meta-analysis of genomewide association studies. Genet Epidemiol 35(7):581-591. 